\title{
Can the transition process from foundation doctor to neurosurgical specialty trainee be improved through "learner-centered induction programs"?
}

\author{
This article was published in the following Dove Press journal: \\ Advances in Medical Education and Practice \\ 16 October 2015 \\ Number of times this article has been viewed
}

\author{
Vikas Acharya' \\ Sami Mansour ${ }^{2}$ \\ Samuel M Amis ${ }^{3}$ \\ Amir Reyahi ${ }^{3}$ \\ 'Department of Neurosurgery, \\ University Hospitals Coventry and \\ Warwickshire, ${ }^{2}$ Warwick Medical \\ School, University of Warwick, \\ Coventry, ${ }^{3}$ Luton and Dunstable \\ University Hospital, Luton, UK
}

\begin{abstract}
The transition period from foundation program doctor to specialty trainee can be difficult for junior doctors. This difficult period often acts as a major obstacle for learning in the workplace. Existing induction programs are commonly seen as inadequate at easing this transition, and therefore, a pilot study intervention was undertaken to assess if the initiation of "learner-centered induction programs" could help improve the confidence, knowledge acquisition, and satisfaction of junior doctors as they begin specialty training in neurosurgery. Ethnographic and anecdotal evidences were collated from junior doctors, specialty trainees, and consultants in order to investigate if further work on this subject would be beneficial. All participants were working in the Department of Neurosurgery at University Hospital Coventry and Warwickshire, Coventry, UK, over a 4-week period in March/April 2015. A review of the relevant literature was also undertaken. This report found that despite the reservations around the increased organizational demands of induction programs of this nature, as well as concerns around a single junior doctor covering the ward alone during the induction period, feedback following the intervention was largely positive. Junior doctors appreciated being taught about their roles and responsibilities from their predecessors as well as deciding among themselves what topics they wanted covering. As a result, the induction sessions tended to focus on clinical skills rather than theoretical knowledge, which most of the junior doctors believed they could cover adequately in their own time. The junior doctors felt that they benefited from learning/ refreshing their relevant practical skills in a safe environment under senior supervision, prior to starting on the wards. Finally, as the induction program was of a greater duration than the traditional half day, they felt they had sufficient time to ask questions and address concerns while "on the job". Overall, "learner-centered induction programs" did appear to show promise in this pilot study with regards to increasing the confidence of junior doctors starting a neurosurgical placement and helped ease the transition process from foundation doctor to specialty trainee in neurosurgery. We believe further work to formalize and quantify these findings using questionnaires and a larger sample group as well as across successive is indicated and may help junior doctor learning and transition processes in future practice.
\end{abstract}

Keywords: induction programs, foundation training, transition processes, junior doctor transitions

\section{Introduction}

Junior doctors undertaking the foundation program in the UK go through a number of transitions as they rotate through different placements and specialties. The transition period for new employees is difficult in any occupation, but there is substantial evidence 
to suggest that this is particularly true for junior doctors going into specialty training. ${ }^{1,2}$ Often they lack confidence in their clinical skills and medical knowledge relevant to the specific specialty, as they have not been exposed to that specialty since medical school, if at all. The junior doctors need to learn where everything is in an alien environment, and many feel that they are not given sufficient pastoral support during this time. They also often need to learn new trust protocols and guidelines if they have moved to a different hospital. This is not only true for newly graduated doctors, but also applicable throughout their whole foundation rotations and into the start of specialty training. Many departments in hospitals around the UK have attempted to address this issue with induction programs for the junior doctors, which usually take the form of lectures and basic generic clinical skills teaching; skills relevant to that particular specialty are rarely included despite it being expected from the doctor on a daily basis. Evidence in the literature suggests that this is usually inadequate and more could be done to improve the induction process. ${ }^{3}$ The significant level of upheaval associated with the transition period from one rotation to another acts as a substantial obstacle to learning in the workplace for junior doctors. If this barrier could be removed with a comprehensive and more learnercentered induction program, junior doctors could potentially start refining skills and building knowledge from the start of the placement, and thus become confident and competent sooner rather than later into their rotation(s).

\section{Induction programs in the literature}

The lack of confidence and preparedness of newly qualified doctors is well documented as the catalyst for the creation of hospital induction programs. Brown et al wrote at length about the difficulties faced by new doctors; a lack of formal guidance and support were common characteristics associated with the first few days in employment. ${ }^{1}$ The authors' findings demonstrate that a lack of support from colleagues leaves many new doctors feeling redundant. It is also noted that progress is further stifled through a general lack of recognition of their contribution and the repetitive nature of common clerical tasks. To defeat the notion that a doctor's 1st year is one to be endured, not enjoyed, the authors assert that new doctors should be placed in an environment where learning is clearly structured and facilitated rather than simply observing on the wards. ${ }^{1}$

Donaghy's study in 2008 developed and evaluated an induction program for junior doctors entering foundation training. ${ }^{3}$ The findings supported the implementation of a program that was standardized, comprehensive, and robust. Significantly, all the trainers who were surveyed thought it was important to have a standardized induction program. "Standardized" is the operative word in this study, as it is later noted that practices not offering a standardized program were especially vulnerable to challenges in the future. The notion that consistency and standardization are key components to structured learning is further elucidated by Nathavitharana's study, advocating the use of an online generic induction package. ${ }^{4}$ The package was developed through multiprofessional collaboration and incorporated the best practices in e-learning with the aim of enhancing patient safety and potentially ending decades of repetitive teaching. The author stressed the importance of making inductions efficient and urged clear guidance on which topics should be covered, in what depth, and how frequently.

It is crucial to acknowledge and understand the psychological upheaval that characterizes transition processes for junior doctors. Both Berridge et al and Evans et al tested the effects on perceived confidence and assessed clinical skills following an induction period. ${ }^{2,5}$ Both studies highlight that junior doctors find their new roles stressful and the transition abrupt. The findings also revealed that junior doctors lacked both confidence and ability with regards to clinical skills. Prior to embarking on training programs, their perceptions about their own abilities were characterized by negativity. Having completed structured inductions, their fears and anxieties were largely assuaged. Both studies advocated the importance of shadowing and clinical skills training during the program. Berridge et al's paper also encouraged the need for full days of shadowing, allowing the additional transmission of experience and generic skills from outgoing junior doctors. ${ }^{2}$ A refinement of the balance between practical learning and academic learning was also encouraged by the authors. The conclusions of both reports were equally explicit and embody the consensus on the implementation of induction programs; they enable learning through the uplifting of confidence and clinical skill-sets and, with ongoing improvement, will form the backbone of learning curricula. From a theoretical stance, it could be seen as "legitimate peripheral participation", involving the junior doctors in the workplace by making the induction relevant to their workplace before they have even started, thereby making them feel already part of the team and prepared for what is about to be experienced. ${ }^{6}$ Though variations are found in the mode of delivery of induction programs, their place as a prerequisite in medical curricula is almost universally supported throughout the literature. This is why it is important to improve induction programs and streamline the transition process from foundation doctor to specialty trainee in neurosurgery. 


\section{Learner-focused induction: trial intervention}

The trial intervention that was assessed during this project involved building substantially on the existing induction program provided by the Neuroscience Department of the University Hospital in Coventry, UK. Input was sought from the junior doctors regarding their previous experiences of induction programs and what improvements they would like to see as well as specific topics they would prefer to be covered relating to neurosurgery. This included practical sessions delivered by senior surgeons on sampling cerebrospinal fluid from external drainage devices, lumbar punctures, and removing wound drains in addition to talks on basic concepts related to managing neurosurgical patients and the commonest complications. A concerted effort was made to give the induction a clear structure that could be reproducible for other specialties and for successive groups of junior doctors. The program was over 3 days, as opposed to a single morning previously, and also included a talk from the juniors imminently leaving the department, regarding their roles and responsibilities on the ward.

\section{Materials and methods}

A prospective cohort observational study was undertaken in the Department of Neurosurgery at University Hospital in Coventry, UK. Informed consent was obtained from all participants involved in the study. No patients were involved in this study and therefore patient consent was not required. Ethical approval was also not formally sought for this project, but departmental approval was granted for the project to be undertaken. The study was longitudinal in nature, as it followed the same group of people over a period of 4 weeks on a weekly basis, with intervals of 1-5 days prior to, during and after the trial intervention. The research/data sample was from clinicians' working in the department during the months of March-May 2015; the participants consisted of five junior doctors (FY2, ST1, ST3, and junior clinical fellow grades), six registrar grade doctors, and six consultants. Vital information was also sought from the four previous junior doctors working in this department during 2014. All data collected were in the form of verbal feedback which stemmed from informal discussions with the participants and were documented as field notes as a part of the ethnographic data collection. Also, a more formal recorded faculty debriefing session after the trial period had ended was used to assess the impact of the learner-centered induction program retrospectively. Participants were allowed to voluntarily opt-in after receiving an information sheet following a detailed description of the projects' plan and intention. This included any doctor working within the department throughout the duration of the project. It focused on clinicians/ participants' working within neurosurgery, specifically foundation and specialty trainee doctors to ensure relevant findings. Our rationale for the above-described methods was stepped from evidence that has been highlighted in various previous studies, commenting on focus groups, participant observation, and ethnographic data having a strong value in accurate findings for educational development, ${ }^{7}$ especially to enable one to gain an insider's narrative and observe practice and change as it happens. ${ }^{8}$ Relevant quotations from the ethnographic evidence collection have been used in the "Results" section to demonstrate the most commonly found issues throughout the pilot study. The overall focus of the informal discussion with the study participants was to assess if this intervention showed promise at improving junior doctor satisfaction and confidence and thus the crucial transition process from foundation doctors to specialty trainees. Furthermore, the pilot study had the more general objective of establishing if this intervention is worth assessing more formally and in-depth with questionnaires to provide more objective, quantifiable data in the future, as well as results from junior doctor work-based assessments to assess if their competency and clinical skills improve with learnercentered induction programs.

\section{Results}

The learner-centered induction program findings from the ethnographic data collected prior to the intervention highlighted that junior doctors largely felt that the past inductions they received did not really benefit or influence daily practice much. The existing induction programs were all lecture based and did not give an opportunity to learn, practice, or improve clinical skills which are unique to this specialty such as basic and common neurosurgical procedures. Junior doctors wanted an induction which would make them more confident with examining and making clinical decisions delivered by senior and experienced clinicians as opposed to the classroom-based theoretical one they received at present. Senior clinicians emphasized the importance of various topics during an induction but acknowledged that induction programs vary depending on who is the main organizer/facilitator and on staff availability. They agreed on the importance of ensuring that sessions were not canceled due to the lack of staff/clinical emergencies and that it should be more evenly distributed to avoid last minute disappointment for the trainees.

Junior and senior doctors largely concurred that a structured and uniform induction program is required to ensure the learning during the induction matches the learning needs of the trainees and may be more applicable over a longer 
period of time (as opposed to the currently allocated half day). Following the trial induction program intervention, the five junior doctors felt that they were happier to see neurosurgical patients having being taught some clinical skills in a safe setting by a senior clinician. The new specialty trainees (ST1 grade) felt that the content was more clinically relevant to their daily work and that the theoretical learning or classroom learning that needed to take place could be done adequately in their own time if necessary. They also now understood their roles and responsibilities better and what was expected of them. Having a fellow junior doctor to teach them decreased anxiety for what they previously thought was "being thrown in at the deep end" without much in the way of support or direction.

During the faculty debriefing session, the six registrars and the six consultants highlighted the difficulty in organizing this more complex induction program, as it meant that more staff members were required but felt that overall "it was beneficial to the learning of the junior doctors". The senior clinicians also felt that this learner-centered induction program should remain uniform and generic for the initial day/phase, and if the learning needs change, then further sessions could be undertaken at further dates but still early enough into the rotation to be classed as an induction. A further concern raised from the seniors' feedback was that for the induction program to take place, a junior doctor had to cover the ward throughout the whole day, similar to an on-call weekend shift. This "overworked that current junior doctor" and had potential implications for patient care.

\section{Discussion}

One of the main findings as a direct result of this report being undertaken was the highlighting of junior doctors' perceptions of the departmental induction program to the faculty. The interviewed junior doctors did not feel that the induction program that was currently in place meets their needs or provides them the information and skills required to benefit their daily practice and prepare them for the transition from foundation doctor to specialty trainee in neurosurgery. It was felt that the induction program did not add much value to what they already knew and therefore did not change their daily work. This finding during this report is supported by medical education literature, where it has been found that junior doctors' lack of preparedness for working can be a direct result of poor support, guidance, and induction within the workplace. ${ }^{1}$ The five junior doctors unanimously mentioned that their previous induction programs did not give them the opportunity to learn, practice, and develop relevant clinical skills. They agreed that an induction program should improve their confidence and ability in clinical decision making and specific procedural skills and felt that this needed to be done practically rather than just through lectures. A study by Evans et al supports this particular finding; they also found that during induction programs, junior doctors lacked confidence and also perceived their clinical skill ability to be lacking. ${ }^{5}$

Upon completion of appropriate induction programs, the negativity toward confidence and perceived ability can change drastically. Brown et al mentioned the importance of giving junior doctors the support in order to make a reasonable start at interpreting and undertaking their roles; this further supports the above-described findings and highlights the need to stress the supporting role that induction programs can have. ${ }^{1}$ Senior clinicians agreed that induction content is important for junior doctors' learning and that induction programs do vary depending on the lead clinician/organizer and staff availability. This suggested that the induction program was not fully learner centered. A further study by Donaghy concluded that induction programs that are not standardized and robust are more likely to face problems in the future, including poor guidance and support for junior doctors. ${ }^{3}$ The feedback from junior and senior doctors alike during this report was that a structured and uniform induction is required, based on the needs of the junior doctors and its relevance for application in daily clinical practice, thus conforming to the evidence base that exists.

Overall, junior doctors and senior doctors agreed that this induction program made the new doctors in the department more confident not only in their roles and responsibilities but also in their clinical abilities. Evidence suggests that if induction programs are clear on what content is to be covered and are efficient, they are increasingly likely to be successful. ${ }^{4}$ This induction was based on what the current junior doctors wanted during their inductions, and so there is a scope for further tailoring of the content and format of the program to fulfill the learner's needs, as the successive groups come through the process and give feedback. This process of continuous feedback and development of the induction program must be central, for it be truly learner centered.

The majority of the interviewed consultants and registrars expressed their increased confidence in the ability of the junior doctors, especially the new specialty trainees following this induction. This can be considered in the context of the social theory aspect of this intervention. An induction program could function as a mean of encouraging "legitimate peripheral participation". ${ }^{6}$ This could not only make 
the junior doctors feel more engaged and more integral part of the workforce before they even begin working but also prepare them for the role that they are about to undertake. By having an induction program that teaches them about the department, prepares them for the role, and teaches them working practices and the unique language of this specialty, they are more likely to be accepted within and seen as a part of this "community of practice" more quickly. ${ }^{6}$

\section{Limitations and further work}

This study has a number of limitations, largely due to its purpose as a pilot study, with the intention that this will lead on to further work in this field. The findings from anecdotal and ethnographic evidence in this pilot study, while providing useful feedback about the potential utility of learner-centered induction programs and possible pitfalls, are of limited but important value. These findings need to be formalized and verified using standardized questionnaires and a larger sample size in order to quantify which proportion of participants agreed with the findings in this study. There would also need to be a control group in future work, undergoing a more traditional 1-day induction program, so that a number of factors such as junior and senior doctor satisfaction, junior doctor competency and clinical skills (from work-based assessment), and junior doctor confidence could then be compared against this control group to more formerly assess any improvements resulting from learnercentered induction programs in neurosurgery. This pilot study was also only conducted across one cycle of induction, so further work across recurrent induction programs to assess whether feedback and subsequent changes further improve the transition process is necessary. As mentioned earlier, a structured and uniform program is needed to ensure success; in the future, a program must be made based on the learner's needs and distributed in advance. This report unfortunately did not have the capacity to do it as its own new program, so it supplemented an existing induction program with more junior doctors' need-based input being taught by experienced/ senior clinicians. Another consideration for future practice would be peer-led teaching as opposed to senior clinician involvement throughout. Evidence suggests that peer-led teaching and learning is highly effective, and therefore, the outgoing junior doctors teaching the incoming junior doctors may be more effective than just using senior clinicians. ${ }^{9}$ This is something that should be looked into not only from the existing evidence point of view but also for future projects being undertaken, as it may be able to further benefit the educational and professional impacts of this intervention beyond what was found during this project.

\section{Acknowledgments}

The authors would like to acknowledge and thank the staff involved in the project within the Neuroscience Department at the University Hospital in Coventry, UK.

\section{Disclosure}

The authors report no conflicts of interest in this work.

\section{References}

1. Brown J, Chapman T, Graham D. Becoming a new doctor: a learning or survival exercise? Med Educ. 2007;41:653-660.

2. Berridge EJ, Freeth D, Sharpe J, Roberts CM. Bridging the gap: supporting the transition from medical student to practising doctor - a two-week preparation program after graduation. Med Teach. 2007;29:119-127.

3. Donaghy F. Induction of foundation program doctors entering general practice. Educ Prim Care. 2008;19:382-388.

4. Nathavitharana K. Online generic induction for doctors in training: an end to repetition? Br J Hosp Med. 2011;72:586-589.

5. Evans DE, Wood DF, Roberts CM. The effect of an extended hospital induction on perceived confidence and assessed clinical skills of newly qualified preregistration house officers. Med Educ. 2004;38:998-1001.

6. Lave J, Wenger E. Situated Learning: Legitimate Peripheral Participation. Cambridge University Press; Cambridge: 1991.

7. Atkinson P, Pugsley L. Making sense of ethnography and medical education. Med Educ. 2005;39:228-235.

8. Barbour R. Making sense of focus groups. Med Educ. 2005;39: 742751.

9. Buckley S, Zamora J. Effects of participation in a cross year peer tutoring program in clinical examination skills on volunteer tutors' skills and attitudes towards teachers and teaching. Med Educ. 2007;7:20.

\section{Publish your work in this journal}

Advances in Medical Education and Practice is an international, peerreviewed, open access journal that aims to present and publish research on Medical Education covering medical, dental, nursing and allied health care professional education. The journal covers undergraduate education, postgraduate training and continuing medical education

\section{Dovepress}

including emerging trends and innovative models linking education, research, and health care services. The manuscript management system is completely online and includes a very quick and fair peer-review system. Visit http://www.dovepress.com/testimonials.php to read real quotes from published authors. 